\section{ACKNOWLEDGMENTS}

The authors wish to thank D. Marcuse for the plot shown in Fig. 1, and J. R. Simpson for the interferograms.

${ }^{1}$ W. A. Gambling, D. N. Payne, H. Matsumura, and R. B. Dyott, "Determination of Core Diameter and Refractive-Index Difference of Single-Mode Fibres by Observation of the Far-Field Pattern," Microwaves Opt. Acoust. 1, 13-17 (1976).

${ }^{2}$ W. A. Gambling, D. N. Payne, H. Matsumura, and R. B. Dyott, "Routine Characterization of Single-Mode Fibres," Electron. Lett. 12, 546-547 (1976).
${ }^{3}$ P. J. B. Claricoats, "Propagation Along Bounded and Unbounded Dielectric Rods," IEE Monog. 409E, (1960).

${ }^{4} \mathrm{E}$. Snitzer, "Cylindrical dielectric waveguide modes," J. Opt. Soc. Am. 51, 491-498 (1961).

${ }^{5}$ A. W. Snyder, "Asymptotic Expressions for Eigenfunctions and Eigenvalues of a Dielectric or Optical Waveguide," IEEE Trans. Microwave Theory Tech. MTT-17, 1103-1137 (1969).

${ }^{6}$ G. W. Tasker, W. G. French, J. R. Simpson, P. Kaiser, and H. M. Presby, "Low loss single-mode fibers with different $\mathrm{B}_{2} \mathrm{O}_{3}-\mathrm{SiO}_{2}$ compositions," Appl. Opt. 17, 1836-1842 (1978).

${ }^{7}$ E. A. J. Marcatili and R. A. Schmeltzer, "Hollow Metallic and Dielectric Waveguides for Long Distance Optical Communications and Lasers," Bell Syst. Tech. J. 43, 1783-1809 (1964).

\title{
Temporal spreading of a pulse propagating in a two-mode optical fiber
}

\author{
Bruno Crosignani* and Charles H. Papas \\ California Institute of Technology, Pasadena, California 91125 \\ Paolo Di Porto \\ Fondazione Ugo Bordoni, Istituto Superiore P. T., Viale Europa, Roma, Italy
}

(Received 4 January 1979; revised 9 June 1979)

\begin{abstract}
The dependence of the temporal width of the impulse response on the length $z$ of a two-mode optical fiber is examined. This quantity, which is proportional to $z$ in the absence of mode coupling and to $z^{1 / 2}$ in the presence of weak random coupling among the guided modes, possesses a different dependence in the case of a deterministic resonant-coupling model, appropriate for describing a rather general class of actual situations. The relevant role played by the coherence time of the signal is demonstrated.
\end{abstract}

\section{INTRODUCTION}

The influence on modal dispersion of coupling among the guided modes of a multimode optical fiber has been examined by many authors ${ }^{1}$ since a possible reduction of the pulse spreading due to this mechanism was first predicted. ${ }^{2}$ The analysis of electromagnetic propagation in a fiber in the presence of mode coupling is usually accomplished in the frame of a statistical theory which deals with quantities such as the powers $P_{n}$ of the individual modes averaged over a suitable ensemble of similar fibers. ${ }^{3}$ This approach allows one to investigate the electromagnetic propagation by means of a set of differential coupled equations describing the evolution of the averaged quantities $\left\langle P_{n}\right\rangle$, which, under certain hypotheses, can be cast in an exactly solvable form. ${ }^{4}$ One of the main results of this theory concerns modal dispersion. More precisely, it turns out that the width of the impulse response exhibits, for a large traveled distance $z$, a squareroot-type dependence on $z$, while the width depends linearly on $z$ in the uncoupled case. ${ }^{5}$ This result implies that for long traveled distances mode coupling reduces dispersion. Reduction of dispersion due to mode coupling has actually been observed. ${ }^{6}$

The statistical approach, which has some intrinsic limitations due to its very nature, ${ }^{7}$ is suitable for dealing with weak-coupling situations such as those arising from the presence of many small unpredictable imperfections in the fiber structure. ${ }^{8}$ However, there are fibers in which mode coupling takes place in such a way that they cannot be considered as typical samples of the ensemble to which the sta- tistical theory applies. This occurs, in particular, in connection with spatially quasiperiodic imperfections giving rise to a resonant coupling of the various modes. In this paper, we wish to examine electromagnetic propagation in a twomode optical fiber possessing this kind of coupling, our main aim being the investigation of the dependence of pulse spreading on length traveled. This spreading turns out to exhibit a behavior that is different from the square-root-type dependence predicted by statistical theory.

The two-mode optical fiber, beyond furnishing the simplest model for treating mode coupling, is interesting per se due to the recent developments of single-mode optical waveguides in which two effective modes with orthogonal polarization are present. 9,10

\section{PROPAGATION IN THE PRESENCE OF RESONANT COUPLING WITH CONSTANT AMPLITUDE}

The propagation of a finite-bandwidth field

$$
\begin{aligned}
& \mathbf{E}(\mathbf{r}, z, t)=\mathbf{E}_{1}(\mathbf{r}) \int c_{1}(z, \omega) e^{i \omega t-i \beta_{1}(\omega) z} d \omega \\
&+\mathbf{E}_{2}(\mathbf{r}) \int c_{2}(z, \omega) e^{i \omega t-i \beta_{2}(\omega) z} d \omega \\
& \equiv \mathbf{E}_{1}(\mathbf{r}) a_{1}(z, t)+\mathbf{E}_{2}(\mathbf{r}) a_{2}(z, t)
\end{aligned}
$$

in a lossless fiber supporting two guided coupled modes is 
governed by the system of equations ${ }^{3,7}$

$$
\begin{aligned}
& \frac{d}{d z} c_{1}(z, \omega)=K_{12}(z) e^{i \Delta(\omega) z} c_{2}(z, \omega) \\
& \frac{d}{d z} c_{2}(z, \omega)=-K_{12}^{*}(z) e^{-i \Delta(\omega) z} c_{1}(z, \omega),
\end{aligned}
$$

which describes the evolution of the mode amplitudes $c_{i}(z, \omega)$. We have indicated with $\mathbf{r}$ and $z$ the transverse and longitudinal coordinates, with $\Delta(\omega)=\beta_{1}(\omega)-\beta_{2}(\omega)$ the difference between the propagation constants of the two unperturbed modes $\mathbf{E}_{1}(\mathbf{r})$ and $\mathbf{E}_{2}(\mathbf{r})$, and with $K_{12}(z)$ the coupling coefficient, which is related to the departure of the fiber structure from the ideal configuration.

If we assume $K_{12}(z)$ to be of the form

$$
K_{12}(z)=2 K \cos (\chi z),
$$

with

$$
\chi=\Delta\left(\omega_{0}\right),
$$

$\omega_{0}$ representing a frequency within the spectral width $\delta \omega$ of the signal, the set of Eqs. (2) becomes

$$
\begin{aligned}
& \frac{d}{d z} c_{1}(z, \omega)=K e^{i\left[\Delta(\omega)+\Delta\left(\omega_{0}\right)\right] z} c_{2}(z, \omega) \\
&+K e^{i\left[\Delta(\omega)-\Delta\left(\omega_{0}\right)\right] z} c_{2}(z, \omega), \\
& \frac{d}{d z} c_{2}(z, \omega)=-K^{*} e^{-i\left[\Delta(\omega)-\Delta\left(\omega_{0}\right)\right] z_{z}} c_{1}(z, \omega) \\
&-K^{*} e^{-i\left[\Delta(\omega)+\Delta\left(\omega_{0}\right)\right] z} c_{1}(z, \omega) .
\end{aligned}
$$

By taking advantage of the rotating wave approximation which, whenever $|K| \ll|\Delta(\omega)|$, allows us to neglect the rapidly oscillating terms of the kind $\exp \left\{ \pm i\left[\Delta(\omega)+\Delta\left(\omega_{0}\right)\right] z\right\}$ that average out to zero over the distance of propagation, the set of Eqs. (5) reduces to

$$
\begin{aligned}
& \frac{d}{d z} c_{1}(z, \omega)=K e^{i \gamma(\omega) z} c_{2}(z, \omega), \\
& \frac{d}{d z} c_{2}(z, \omega)=-K^{*} e^{-i \gamma(\omega) z} c_{1}(z, \omega),
\end{aligned}
$$

with $\gamma(\omega)=\Delta(\omega)-\Delta\left(\omega_{0}\right)$. The set of Eqs. (6) can be easily solved and the resulting expressions for $c_{1}(z, \omega)$ and $c_{2}(z, \omega)$ inserted into Eq. (1), thus giving'11

$$
\begin{aligned}
a_{1}(z, t)= & \exp \left(-i \Delta\left(\omega_{0}\right) z / 2\right) \\
& \times \int_{-\infty}^{+\infty} \exp \left\{-i\left[\beta_{1}(\omega)+\beta_{2}(\omega)\right] z / 2+i \omega t\right\} \\
& \times\left\{A \exp \left[i|K| z+i\left(\omega-\omega_{0}\right)^{2} z / 8|K| \tilde{v}^{2}\right]\right. \\
& \left.+B \exp \left[-i|K| z-i\left(\omega-\omega_{0}\right)^{2} z / 8|K| \tilde{v}^{2}\right]\right\} d \omega
\end{aligned}
$$

and

$$
\begin{aligned}
& a_{2}(z, t)= i \exp \left[-i \phi+i \Delta\left(\omega_{0}\right) z / 2\right] \\
& \times \int_{-\infty}^{+\infty} \exp \left\{-i\left[\beta_{1}(\omega)+\beta_{2}(\omega)\right] z / 2+i \omega t\right\} \\
& \times\left\{A \exp \left[i|K| z+i\left(\omega-\omega_{0}\right)^{2} z / 8|K| \tilde{v}^{2}\right]\right. \\
&\left.\quad-B \exp \left[-i|K| z-i\left(\omega-\omega_{0}\right)^{2} z / 8|K| \tilde{v}^{2}\right]\right\} d \omega .
\end{aligned}
$$

Here,

$$
\begin{aligned}
& A=(1 / 2)\left[c_{1}(0, \omega)-i e^{i \phi} c_{2}(0, \omega)\right], \\
& B=(1 / 2)\left[c_{1}(0, \omega)+i e^{i \phi} c_{2}(0, \omega)\right],
\end{aligned}
$$

where $\exp (i \phi)=K /|K|$ and

$$
1 / \tilde{v}=1 / V_{1}-1 / V_{2},
$$

$V_{i}^{1}=\left(d \beta_{i} / d \omega\right)_{\omega=\omega_{0}}$ indicating the inverse of the group velocity of the $i$ th unperturbed mode. Furthermore, we have assumed

$$
\delta \omega \ll|K| \tilde{v}
$$

and

$$
\Delta(\omega) \simeq \Delta\left(\omega_{0}\right)+\left(\omega-\omega_{0}\right) / \tilde{v},
$$

$\delta \omega$ being the source bandwidth.

The electromagnetic powers $P_{1}(z, t)$ and $P_{2}(z, t)$ carried by modes 1 and 2, whose behavior determines the pulse shape, are proportional to $\left\langle\left|a_{1}(z, t)\right|^{2}\right\rangle_{\text {av }}$ and $\left\langle\left|a_{2}(z, t)\right|^{2}\right\rangle_{\text {av }}$, where the symbol \langle\rangle$_{a v}$ indicates the averaging operation over the fluctuations of the source exciting the fiber. In order to determine $\left\langle\left|a_{1}(z, t)\right|^{2}\right\rangle_{\text {av }}$ and $\left\langle\left|a_{2}(z, t)\right|^{2}\right\rangle_{\mathrm{av}}$, it is necessary to evaluate the quantities $\left\langle c_{1}(0, \omega) c_{1}^{*}\left(0, \omega^{\prime}\right)\right\rangle_{\mathrm{av}},\left\langle c_{2}(0, \omega) c_{2}^{*}\left(0, \omega^{\prime}\right)\right\rangle_{\mathrm{av}}$, and $\left\langle c_{1}(0, \omega) c_{2}^{*}\left(0, \omega^{\prime}\right)\right\rangle_{\text {av }}$ as functions of the source bandwidth and pulse duration. By assuming a pulse of Gaussian shape and width $T_{p}$ injected into the fiber by a source possessing a coherence time $T_{c}=2 \pi / \delta \omega$, one has ${ }^{11}$

$$
\begin{gathered}
\left\langle c_{1}(0, \omega) c_{1}^{*}\left(0, \omega^{\prime}\right)\right\rangle_{\mathrm{av}}=\left\langle c_{1}\left(0, \omega_{0}\right) c_{1}^{*}\left(0, \omega^{\prime}\right)\right\rangle_{\mathrm{av}} \\
\times \exp \left(-\Omega^{2} T_{1}^{2} / 4-\Omega^{\prime 2} T_{1}^{2} / 4+\Omega \Omega^{\prime} T_{2}^{2} / 2\right) \equiv F\left(\Omega, \Omega^{\prime}\right),
\end{gathered}
$$

where

$$
T_{1}^{2}=T_{P}^{2}\left(T_{p}^{2}+T_{c}^{2}\right) /\left(2 T_{p}^{2}+T_{c}^{2}\right)
$$

and

$$
T_{2}^{2}=T_{p}^{4} /\left(2 T_{p}^{2}+T_{c}^{2}\right),
$$

with $\Omega=\omega-\omega_{0}$ and $\Omega^{\prime}=\omega^{\prime}-\omega_{0}$.

With the help of Eqs. (7), (8), and (13) we can write, after assuming for the sake of simplicity that $c_{1}(0, \omega)=c_{2}(0, \omega)$,

$$
\begin{aligned}
\left\langle\left|a_{1}(z, t)\right|^{2}\right\rangle_{\mathrm{av}}=\int_{-\infty}^{+\infty} d \Omega \int_{-\infty}^{+\infty} d \Omega^{\prime} e^{i\left(\Omega-\Omega^{\prime}\right) t} & \\
& \times H\left(z, \Omega, \Omega^{\prime} ; K\right) F\left(\Omega, \Omega^{\prime}\right),
\end{aligned}
$$

where

$$
\begin{aligned}
H\left(z, \Omega, \Omega^{\prime} ; K\right)= & (1 / 2)\left[(1+\sin \phi) e^{i\left(\Omega^{2}-\Omega^{\prime 2}\right) \tau^{2}(z)}\right. \\
& +(1-\sin \phi) e^{i\left(\Omega^{\prime 2}-\Omega^{2}\right) \tau^{2}(z)} \\
& +i \cos \phi e^{-2 i|K| z} e^{-i\left(\Omega^{2}+\Omega^{\prime 2}\right) \tau^{2}(z)} \\
& \left.-i \cos \phi e^{2 i|K| z} e^{i\left(\Omega^{2}+\Omega^{\prime 2}\right) \tau^{2}(z)}\right]
\end{aligned}
$$

and

$$
\tau^{2}(z)=z / 8|K| \tilde{v}^{2},
$$

the same expression being valid for $\left\langle\left|a_{2}(z, t)\right|^{2}\right\rangle_{\mathrm{av}}$ but for the substitution $\cos \phi \rightarrow-\cos \phi$.

By performing the double integral appearing in Eq. (16), one finally obtains 


$$
\begin{aligned}
\left\langle\left|a_{1,2}(z, t)\right|^{2}\right\rangle_{\mathrm{av}}=\frac{4 \pi\left\langle c_{1}\left(0, \omega_{0}\right) c_{1}^{*}\left(0, \omega_{0}\right)\right\rangle_{\mathrm{av}}}{T_{3}\left(T_{0}^{2}+16 \tau^{4}(z) / T_{3}^{2}\right)^{1 / 2}} & \exp \left\{-2 t^{\prime 2} /\left[T_{0}^{2}+16 \tau^{4}(z) / T_{3}^{2}\right]\right\} \pm \frac{4 \pi \cos \phi\left\langle c_{1}\left(0, \omega_{0}\right) c_{1}^{*}\left(0, \omega_{0}\right)\right\rangle_{\mathrm{av}}}{\left\{\left[T_{3}^{2} T_{0}^{2}-16 \tau^{4}(z)\right]^{2}+64 T_{1}^{4} \tau^{4}(z)\right\}^{1 / 4}} \\
\times \exp (- & \left.2 t^{\prime 2}\left[T_{3}^{4} T_{0}^{2}+16 T_{0}^{2} \tau^{4}(z)\right] /\left\{\left[T_{3}^{2} T_{0}^{2}-16 \tau^{4}(z)\right]^{2}+64 T_{1}^{4} \tau^{4}(z)\right\}\right) \\
\times & \times \sin \left(2 t^{\prime 2}\left[4 T_{3}^{4} \tau^{2}(z)+64 \tau^{6}(z)\right] /\left\{\left[T_{3}^{2} T_{0}^{2}-16 \tau^{4}(z)\right]^{2}+64 T_{1}^{4} \tau^{4}(z)\right\}+2|K| z+\psi / 2\right),
\end{aligned}
$$

where

$$
t^{\prime}=t-z / V
$$

with

$$
\begin{gathered}
1 / V=\left(1 / V_{1}+1 / V_{2}\right) / 2, \\
t_{3}^{2}=T_{1}^{2}-T_{2}^{2}=T_{p}^{2} T_{C}^{2} /\left(2 T_{p}^{2}+T_{C}^{2}\right), \\
T_{0}^{2}=T_{1}^{2}+T_{2}^{2}=T_{p}^{2},
\end{gathered}
$$

and

$$
\psi=\arg \left[T_{3}^{2} T_{0}^{2}-16 \tau^{4}(z)-8 i T_{1}^{2} \tau^{2}(z)\right] .
$$

The significant result contained in Eq. (19) is that the powers $P_{1}(z, t)$ and $P_{2}(z, t)$ exhibit the same space-time evolution, traveling with a bulk velocity $V$ and undergoing a broadening which, for $z$ sufficiently large $(z>\bar{z}, \bar{z}$ being defined through the relation $\left.\tau^{2}(\bar{z})=T_{P} T_{3} / 4\right)$, is proportional to the traveled length itself.

Actually, it is the first term on the right-hand side of Eq. (19) that plays the leading role for what concerns broadening, since $T_{3}<T_{p}$ [see Eqs. (22) and (23)]. In practice, $T_{c} \ll T_{p}$, so that $T_{3} \simeq T_{c} / \sqrt{2} \ll T_{p}$. Accordingly, the temporal width $T_{p}(z)$ of the pulse can be evaluated as

$$
T_{p}(z)=z /\left(2|K| \tilde{v}^{2} T_{c}\right)^{1 / 2} \text { for } z>\bar{z},
$$

so that the pulse temporal width behaves linearly with $z$, as in the absence of mode coupling.

The width $T_{p}(z)$ can be easily shown to be much smaller than $T=z / \tilde{v}$ relative to the noncoupling case, that is, to the difference between the group delays of the two unperturbed modes. As a matter of fact, one has

$$
T_{p}(z)=(T / \sqrt{2})(\delta \omega / 2 \pi|K| \tilde{v}),
$$

so that, according to Eq. (11), $T_{p}(z) \ll T$. This fact confirms the positive role played by coupling in reducing dispersion. Thus, the influence of mode coupling on pulse broadening is only of a quantitative nature in our deterministic model, while, in the framework of the statistical theory, mode coupling results in a $z$ dependence of the pulse width of the square-root type. This latter kind of dependence can be accounted for in an intuitive way by taking advantage of the Central Limit Theorem in connection with the random "jumps" of each photon from one mode to the other, ${ }^{12}$ while no random process occurs in our model.

We wish to conclude this section by noting the relevance of the coherence time $T_{c}$ of the source [provided by Eq. (11) is verified] in determining the pulse spreading in our case, while $T_{c}$ (or, equivalently, the source bandwidth) does not influence the pulse shape according to the conclusions of the statistical approach. ${ }^{3}$ This is directly related to the basic hypothesis underlying the statistical theory, according to which the field amplitudes do not vary appreciably over the small distance after which the mode-coupling random imperfections decorrelate, leading to a system of coupled equations involving only the mode powers $\left\langle P_{n}\right\rangle .^{3}$ On the contrary, coherence effects are preserved in our situation due to the periodicity of the coupling coefficient, a fact that does not allow consideration of our fiber as a typical sample of the statistical ensemble.

\section{INFLUENCE OF VARIATIONS OF COUPLING AMPLITUDE AND PERIODICITY}

A more general coupling model can be obtained by letting the amplitude $K$ and the periodicity $\chi$ of $K_{12}(z)$ to assume different constant values $K_{i}$ and $\chi_{i}$ over different fiber regions (possessing, in general, different lengths $L_{i}$ ). In order to preserve the resonance condition, we assume the existence of frequencies $\omega_{i}$ lying inside the bandwidth $\delta \omega$ such that

$$
\chi_{i}=\beta_{1}\left(\omega_{i}\right)-\beta_{2}\left(\omega_{i}\right) .
$$

From an analytical point of view, the problem of pulse propagation in this structure can be easily solved by observing that, due to the form of Eq. (16), the quantity

$$
H\left(L_{1}, \Omega, \Omega^{\prime} ; K_{1}\right) F\left(\Omega, \Omega^{\prime}\right)
$$

furnishes the "boundary condition" for deriving the solution in the second region, which reads

$$
\begin{aligned}
\left\langle\left|a_{1}(z, t)\right|^{2}\right\rangle_{\mathrm{av}} & =\int_{-\infty}^{+\infty} d \Omega \int_{-\infty}^{+\infty} d \Omega^{\prime} e^{i\left(\Omega-\Omega^{\prime}\right) t} \\
& \times H\left(z_{2}, \Omega, \Omega^{\prime} ; K_{2}\right) H\left(L_{1}, \Omega, \Omega^{\prime} ; K_{1}\right) F\left(\Omega, \Omega^{\prime}\right),
\end{aligned}
$$

for $0 \leq z_{2} \leq L_{2}$ and $z=L_{1}+z_{2}$. More in general, one has

$$
\begin{aligned}
& \left\langle\left|a_{1}(z, t)\right|^{2}\right\rangle_{\mathrm{av}}=\int_{-\infty}^{+\infty} d \Omega \int_{-\infty}^{+\infty} d \Omega^{\prime} e^{i\left(\Omega-\Omega^{\prime}\right) t} \\
& \quad \times H\left(z_{n}, \Omega, \Omega^{\prime} ; K_{n}\right) H\left(L_{n-1}, \Omega, \Omega^{\prime} ; K_{n-1}\right) \\
& \quad \times H\left(L_{n-2}, \Omega, \Omega^{\prime} ; K_{n-2}\right) \times \cdots H\left(L_{1}, \Omega, \Omega^{\prime} ; K_{1}\right) F\left(\Omega, \Omega^{\prime}\right), \\
& \text { for } 0 \leq z_{n} \leq L_{n} \text { and } z=L_{1}+L_{2}+\cdots+L_{n-1}+z_{n} .
\end{aligned}
$$

The right-hand side of Eq. (30) is the sum of a number of terms of the kind

$$
\int_{-\infty}^{+\infty} d \Omega \int_{-\infty}^{+\infty} d \Omega^{\prime} \exp \left(i a \Omega^{2}+i b \Omega^{\prime 2}\right)
$$

$$
\times \exp \left[i\left(\Omega-\Omega^{\prime}\right)(t-z / V)\right] F\left(\Omega, \Omega^{\prime}\right),
$$

where $a$ and $b$ are linear combinations of the quantities $\tau^{2}\left(L_{1}\right)$, $\tau^{2}\left(L_{2}\right), \ldots, \tau^{2}\left(z_{n}\right)$ with coefficients +1 and -1 in all possible combinations. By recalling Eq. (13), the double integral appearing in Eq. (31) can be explicitly evaluated. The most relevant contributions to $\left\langle\left|a_{1}(z, t)\right|^{2}\right\rangle_{\text {av }}$ come from the terms for which

$$
a=-b= \pm\left[\tau^{2}\left(L_{1}\right)+\tau^{2}\left(L_{2}\right)+\cdots+\tau^{2}\left(z_{n}\right)\right],
$$

which evolve in the form of a pulse moving with a velocity $V$ and possessing a temporal width $T_{p}(z)=T_{p}\left(L_{1}+L_{2}+\cdots\right.$ 


$$
\begin{aligned}
& \left.+L_{n-1}+z_{n}\right) \text { given by } \\
& T_{p}(z) \\
& \quad=\frac{L_{1} /\left|K_{1}\right|+L_{2} /\left|K_{2}\right|+\cdots+L_{n-1} /\left|K_{n-1}\right|+z_{n} /\left|K_{n}\right|}{\sqrt{2} \tilde{v}^{2} T_{c}} .
\end{aligned}
$$

Equation (33) generalizes Eq. (25) but assures a linear dependence of $T_{p}(z)$ on the traveled length only inside each single fiber region with a given value of $|K|$.

\section{CONCLUSIONS}

We have investigated the broadening of a pulse propagating in a two-mode fiber, made up of a number of regions in each of which a spatially periodic resonant coupling with constant amplitude is present. This kind of optical waveguide does not belong to the statistical ensemble considered in the randomcoupling theory, so that electromagnetic propagation cannot be treated by means of coupled-power-equations, and the mode-amplitude evolution has to be explicitly investigated. As a consequence, the temporal broadening of the pulse turns out to be related to the source coherence time, while it does not exhibit the dependence on the square root of the fiber length predicted by the statistical approach.

\section{ACKNOWLEDGMENT}

This research was supported by the U.S. Army Research Office under Contract No. DAAG29-77-G-0130.
* On leave of absence from Fondazione Ugo Bordoni, Roma, Italy.

${ }^{1}$ See, for example, R. Olshansky, "Mode coupling effects in gradedindex optical fibers," Appl. Opt. 14, 935-945 (1975) and bibliography therein.

${ }^{2}$ S. D. Personick, "Time dispersion in dielectric waveguides," Bell Syst. Tech. J. 50, 843-859 (1971).

${ }^{3} \mathrm{D}$. Marcuse, Theory of Dielectric Optical Waveguides (Academic, New York, 1974).

${ }^{4} \mathrm{D}$. Gloge, "Optical power flow in multimode fibers," Bell Syst. Tech. J. 51, 1767-1783 (1972).

${ }^{5}$ This result holds true for a single fiber; for the situation of many fibers jointed together see, for example, M. Eve, "Multipath time dispersion theory of an optical network," Opt. Quantum Electron. 10, 41-51 (1978).

${ }^{6}$ E. L. Chinnock, L. G. Cohen, W. S. Holden, R. D. Standley, and D. B. Keck, "The length dependence of pulse spreading in the CGW-Bell-10 optical fiber," Proc. IEEE (Lett.) 61, 1499-1500 (1973).

${ }^{7}$ For a detailed discussion of this point, see B. Crosignani, P. Di Porto, and C. H. Papas, "Theory of time-dependent propagation in multimode lightguides," J. Opt. Soc. Am. 67, 1300-1307 (1977).

${ }^{8}$ The weak-coupling hypothesis also underlies the results of the ray theory; see, for example, J. Arnaud and M. Rousseau, "Ray theory of randomly modulated optical fibers," Opt. Lett. 3, 63-65 (1978).

${ }^{9} \mathrm{~S}$. C. Rashleigh and R. Ulrich, "Polarization mode dispersion in single-mode fibers," Opt. Lett. 3, 60-62 (1978).

${ }^{10}$ R. H. Stolen, V. Ramaswamy, P. Kaiser, and W. Pleibel, "Linear polarization in birefringent single-mode fibers," Appl. Phys. Lett. 33, 699-701 (1978).

${ }^{11 B}$ B. Crosignani, P. Di Porto, and C. H. Papas, "Modal dispersion in lightguides in the presence of strong coupling," J. Opt. Soc. Am. 68, 1586-1591 (1978).

${ }^{12} \mathrm{~S}$. Kawakami and M. Ikeda, "Transmission characteristics of a two-mode optical waveguide," IEEE J. Quantum Electron. QE-14, 608-614 (1978).

\title{
Exact expressions for scalar modal eigenvalues and group delays in power-law optical fibers
}

\begin{abstract}
Colin Pask
Department of Applied Mathematics, Research School of Physical Sciences, Australian National University, Canberra, ACT 2601, Australia

(Received 9 March 1979)

Considerable use is made of power-law refractive-index profiles in fiber optics. These profiles have simple scaling properties that are exploited in this paper to obtain formulas for the exact modal propagation constants and group delays. When an infinite profile is assumed, the results of the exact, WKB, and geometric optics theories are all shown to agree. This conclusion remains valid when linear material dispersion is included. The effect of the cladding is discussed. The results are correct for noncircular fibers of power-law type.
\end{abstract}

\section{INTRODUCTION}

Various investigations culminating in the Gloge and Marcatili 1973 paper $^{1}$ established the power-law refractive index profile as one of major significance in fiber optics. The optimization of this profile, the importance of deviations from it and the influence of material dispersion in the optimization process have claimed the attention of a large number of theorists. On the experimental side, power-law profiles have given the fiber fabricator something to aim for and also have provided a reference frame for analyzing certain experimental results. Our concern in this paper is with the basic theoretical analysis of power-law fibers.
Light is described by a vector wave function that satisfies Maxwell's electromagnetic equations. ${ }^{2}$ In general, these equations cannot be solved but, when core and cladding refractive indices differ slightly, as is the usual case, the fields can be constructed from scalar wave functions $\psi .2,3$ For mode $j$ in a fiber uniform along its length and aligned with the $z$ axis, the function $\psi_{j}$ is obtained by solving

$$
\left[\nabla^{2}+\left(n^{2} k^{2}-\beta_{j}^{2}\right)\right] \psi_{j}=0,
$$

plus appropriate boundary conditions. In Eq. (1), $\beta_{j}$ is the modal propagation constant [modal field $z$ dependence is $\left.\exp \left(i \beta_{j} z\right)\right], k$ is the wave number $2 \pi / \lambda$ where $\lambda$ is the wave- 